\title{
Comportamiento de los usuarios en la página de resultados de los buscadores. Un estudio basado en eye tracking
}

\author{
Por Mari-Carmen Marcos y Cristina González-Caro
}

\begin{abstract}
Resumen: Se presenta un estudio realizado sobre las páginas de resultados de Google y Yahoo! utilizando la técnica de eye tracking. 58 participantes han tratado de resolver tareas que llevaban implícita una intención informacional, navegacional, transaccional o multimedia. Las sesiones se han grabado con eye tracker con el fin de comprobar si la intención de las consultas afecta a la forma en que el usuario explora visualmente la página de resultados de un buscador. Para ello hemos estudiado las fijaciones de la vista en el título, snippet, url e imágenes de los tres primeros resultados y en los tres primeros enlaces patrocinados. Los resultados muestran una relación entre la intención de búsqueda del usuario y su comportamiento en la exploración de la página de resultados. Conocer este comportamiento resulta importante para los buscadores, que pueden mejorar sus páginas de resultados en función de la intención de las consultas de los usuarios.
\end{abstract}

Palabras clave: Recuperación de información, Buscadores, Intención del usuario, Eye tracking, Comportamiento de los usuarios.

Title: User behavior in the search engines results page: a study based on the eye tracking technique

Abstract: A study based on Google and Yahoo! page results using eye tracker technique is presented. Participants $(n=58)$ attempted informational, navigational, transactional or multimedia tasks. Sessions were recorded with an eye tracker to determine whether the intention behind queries affects the way people browse the results page. Eye fixations in title, snippet, url and images were analyzed in the three first organic and sponsored results. In general terms, the results demonstrate that a relationship exists between the users' intention and their behavior when they browse the results page. Knowing this behavior is important for search engine designers because they can improve their results pages depending on the users' query intentions.

Keywords: Information retrieval, Search engines, User intention, Eye tracking, User behavior.

Marcos, Mari-Carmen; González-Caro, Cristina. "Comportamiento de los usuarios en la página de resultados de los buscadores. Un estudio basado en eye tracking”. El profesional de la información, 2010, julio-agosto, v. 19, n. 4, pp. 348358.

DOI: $10.3145 /$ epi.2010.jul.03

\section{Introducción}

Existen distintas disciplinas y profesiones que estudian los buscadores de internet. Por un lado la SEO (search engine optimization) trata de mejorar la posición de las webs en la lista de resultados de los buscadores (Codina, Marcos, 2005); por otro, la analítica web estudia los movimientos del usuario dentro de la web a la que accede (Kaushik, 2007, 2009). Lo que ocurre cuando el usuario se encuentra frente a la página de resultados del buscador (SERP, search engine results page) no lo cubre ninguna de ellas, sino la HCI ( $h u$ man computer interaction) con la finalidad de proponer cambios para una mejor experiencia de uso de estas herramientas, y la IR (information retrieval) para que los motores de búsqueda sean más eficientes (figura 1).

La forma en que los buscadores han presentado 


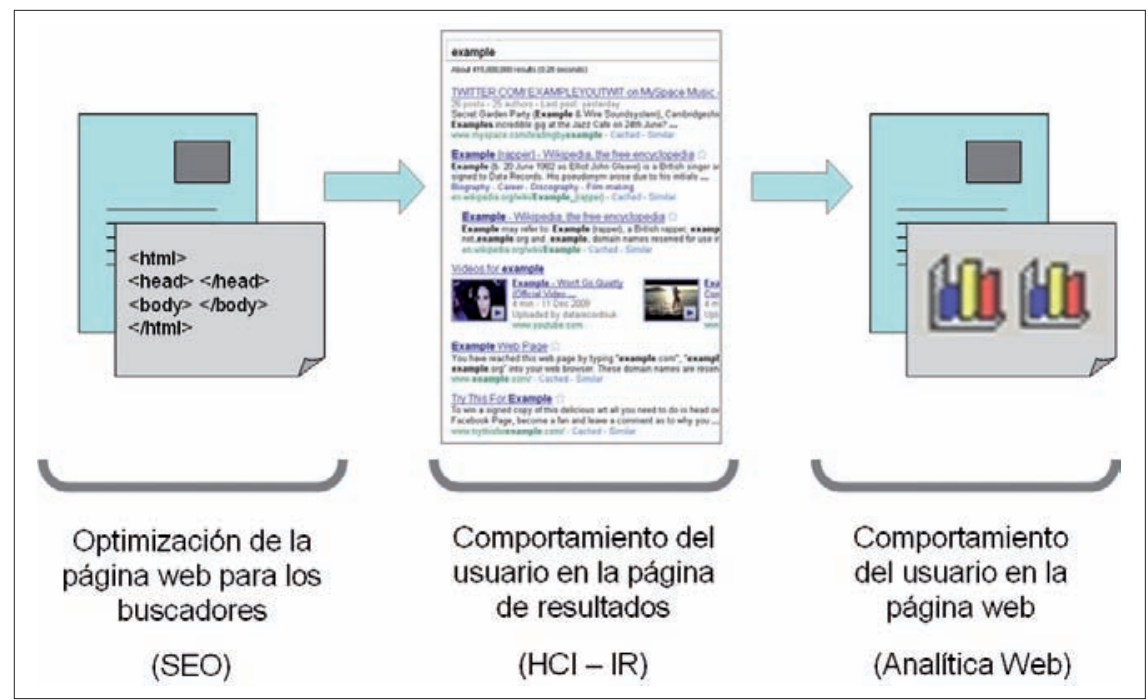

Figura 1. Contexto del estudio del comportamiento de los usuarios en la SERP

tradicionalmente las páginas web recuperadas ante una consulta es el listado. Los listados, ordenados en función de la relevancia de cada página y de otros criterios para los que se aplican complejos algoritmos, presentan en pocas líneas una información que debe servir a los usuarios para determinar si la web es o no de su interés: el título, un fragmento de texto (snippet) con la información más cercana a las palabras de la consulta, y el url; en ocasiones también se muestra una imagen o un mapa. Por tanto la decisión del usuario a la hora de clicar está basada en muy poca información. A estos resultados -llamados orgánicos-, se añaden los enlaces patrocinados que, con un formato similar a los anteriores, se sitúan en la parte superior o en el lateral derecho de la pantalla.

Ante esta variedad de tipos de resultados y de información sobre los mismos, resulta interesante analizar el comportamiento de los usuarios en las SERP para ver si existen patrones que ayuden a mejorar el proceso de recuperación de información. Así como en SEO y en analítica web se utilizan los logs de tráfico, para estudiar el comportamiento de las personas en una página en particular es necesario otro tipo de técnicas, ya que no se dejan marcas de navegación. Éstas son el seguimiento de la mirada y de los movimientos del ratón.

El escrutinio de los resultados del buscador no necesariamente implica uso del ratón hasta que se toma la decisión de clicar, por lo que es más conveniente analizar la mirada. Para ello se puede utilizar la tecnología de eye tracking y obtener datos del número de veces que las personas miran determinada parte de la página (número de fijaciones) y del tiempo que dura esa mirada (duración de las fijaciones) (Van Gompel, Fischer, Murria y Hill, 2007).

En este estudio tratamos de descubrir si existen patrones de comportamiento determinantes para diferen- tes tipos de intenciones. Para ello partimos de la propuesta de Broder (2002), quien distingue tres tipos de consultas en función de las tres intenciones que los usuarios pueden tener a priori en sus búsquedas:

- Informacional: el usuario quiere obtener información, por ejemplo el teléfono de un hospital, la temperatura de una ciudad o la biografía de un cantante.

- Navegacional: quiere llegar a un sitio web determinado, por ejemplo el de una universidad en la que quiere estudiar, el de la empresa para la que va a hacer una entrevista de trabajo o el de un diario que le gustaría leer.

- Transaccional: quiere realizar una acción, por ejemplo descargar un software, comprar un billete de avión o solicitar un certificado de empadronamiento.

A esta clasificación hemos añadido un tipo más. Se trata de las búsquedas multimedia, que son aquellas que tienen como finalidad ver una fotografía o un vídeo.

\section{"Se consideran cuatro tipos de consultas: informacional, navegacional, transaccional y multimedia"}

Nuestra hipótesis de partida es que en función de la intención de búsqueda del usuario, la atención se centra más en una determinada parte de la información que aparece en pantalla en la página de resultados. Creemos que la parte que más se observe variará en función de la intención de búsqueda de esta forma:

- Informacional: el usuario leerá con más atención los snippets para saber si la página web puede responder a lo que busca. No dará mucha importancia a los anuncios laterales, pero posiblemente sí a los superiores, sobre todo en el caso de usuarios noveles que desconocen que los primeros resultados presentados suelen ser anuncios.

- Navegacional: el usuario leerá con atención el título de la página para ver si responde al nombre de la empresa, institución o marca que busca; y en caso de ser un usuario avanzado leerá también el url que aparece para asegurarse de que se trata de la página oficial.

- Transaccional: el usuario prestará más atención a los enlaces patrocinados que en los casos anteriores, en 
particular el título del anuncio; y en los resultados orgánicos revisará tanto el título como el snippet, pero no tanto como en el caso de búsquedas informacionales.

- Multimedia: el usuario focalizará su atención casi exclusivamente en la imagen y no en el resto de información de la página de resultados.

Si se logra ver una relación entre la intencionalidad y la forma de mirar la pantalla habremos avanzado un paso en la línea de investigación de la predicción de las intenciones de los usuarios. Esto servirá para mejorar las interfaces de resultados en los buscadores, siempre y cuando otros trabajos demuestren la fiabilidad de estas predicciones.

\section{Trabajos previos}

El primer estudio publicado que utiliza la técnica de eye tracking para analizar el comportamiento de los usuarios en las SERPs es el de Granka, Joachims y Gay (2004), pero no tienen en cuenta la intención de búsqueda del usuario.

Aula, Majaranta y Räihä (2005) se centraron en estudiar los estilos de búsqueda de los usuarios a la hora de evaluar los resultados obtenidos. Por su parte Rele y Duchowski (2005) evaluaron dos tipos de interfaces de páginas de resultados y usaron dos clases de búsqueda (informacional y navegacional), pero se centraron en el análisis de la usabilidad de las interfaces y no en el del comportamiento del usuario para los dos tipos de búsqueda. El informe de Enquiro (Hotchkiss, Alston y Edwards, 2006) demuestra que prácticamente todas las miradas se concentran en los tres primeros resultados orgánicos, siendo muy mínima la observación de los anuncios y prácticamente nula la de resultados por debajo del tercero, de ahí que buena parte de los esfuerzos de los webmasters se dedique a tratar de obtener uno de esos primeros puestos. El estudio denomina a esta área clave el "triangulo de oro", y demuestra que la ubicación de los resultados es importante para su posterior selección por parte de los usuarios.

Guan y Cutrell (2007) profundizan en el análisis del comportamiento de los usuarios en función de la ubicación de los resultados relevantes dentro de las SERPs y encuentran diferencias entre las consultas informacionales y navegacionales. Los mismos autores (Cutrell y Guan, 2007) realizan otro experimento en el que estudian cómo afecta el comportamiento de los usuarios la modificación del snippet y del título; y descubren que un snippet más extenso favorece las consultas informacionales y uno más breve las navegacionales. En ninguno de los dos trabajos abordan las consultas transaccionales.

Por otro lado el informe GEA Internet project consulting (2009) usa eye tracking para estudiar las SERPs, pero no entra en detalle sobre la zona de los resultados que se observa con mayor atención en función del tipo de consulta. Lorigo et al. (2008) realizan un estudio en el que por primera vez se usan dos buscadores, Google y Yahoo! y analizan si el grado de experiencia del usuario con un determinado buscador influye en su comportamiento en la SERP cuando se trata de tareas con intención informacional.

Terai et al. (2008) estudian las diferencias entre consultas de tipo informacional y transaccional. Se centran en la secuencia de navegación del usuario en la SERP pero no llegan al detalle de las áreas de interés evaluadas ni profundizan en el comportamiento frente a los enlaces patrocinados, tan importantes en las búsquedas con intención transaccional. Finalmente, Granka, Feusner y Lorigo (2008) presentan un completo estado de la cuestión sobre la aplicación de la técnica de eye tracking en las páginas de resultados de los buscadores, detallando las distintas métricas que pueden utilizarse y las principales aportaciones de los trabajos publicados.

\section{"Una de las novedades de este trabajo es el estudio de la relación entre los patrones de mirada y la intencionalidad que hay tras las consultas"}

En el presente estudio tratamos de descubrir si existen patrones de comportamiento determinantes para diferentes tipos de intenciones, y para ello introducimos dos novedades respecto a la bibliografía anterior:

- Un mayor nivel de detalle, para lo que se estudian con eye tracker las distintas zonas de cada resultado en la SERP, denominadas áreas de interés (AoIs).

- La relación entre los patrones de mirada y la intencionalidad que hay tras las consultas.

Los resultados obtenidos nos darán una guía de comportamiento de los usuarios en la página de resultados en función de las intenciones de búsqueda. Ello será de utilidad para creadores de páginas web y para responsables de buscadores. Los primeros, para crear sus páginas considerando cómo se explora lo poco que los buscadores muestran de ellas en la lista de resultados, y los segundos para diseñar la página de resultados acorde a la intención de las consultas.

\section{Diseño del experimento}

Se ha utilizado la técnica de seguimiento de la mirada (eye tracking) con un grupo de personas que 
habitualmente utilizan buscadores web. Presentamos a continuación los motores de búsqueda utilizados, el tipo de consultas, el tipo de participantes seleccionados para realizar las pruebas, las variables contempladas, cómo se preparó el software y cómo se llevaron a cabo los tests con usuarios.

\subsection{Buscadores}

Para las consultas textuales (informacionales, navegacionales y transaccionales) se han utilizado Google. es y Yahoo.es por ser los dos más utilizados actualmente en España. Para las consultas multimedia se han usado Google y Yahoo! en sus buscadores específicos de imágenes y vídeos.

\subsection{Participantes}

Durante el mes de mayo de 2009 se testeó a 58 usuarios (RealEyes, 2007) con edades comprendidas entre los 18 y los 55 años. Estaban repartidos así: 25 hombres (43\%) y 33 mujeres (57\%). El $80 \%$ de ellos se situaba entre los 20 y los 30 años. Todos eran usuarios habituales de internet y tenían estudios superiores terminados o en curso. A cada uno de ellos se le citó de forma individual. La duración de las pruebas fue de 30 minutos por participante.

\subsection{Tareas}

Se diseñó una batería de 22 consultas que incluían las cuatro intenciones: 10 informacionales, 3 navegacionales, 4 transaccionales y 5 multimedia. La decisión de tomar un número diferente de tareas en cada tipo está basada en los datos que aporta Broder (2002) a partir de una encuesta realizada a los usuarios de $\mathrm{Al}$ tavista y el análisis de $\operatorname{los} \log s$, donde se revela que más de la mitad de las consultas que se realizan tienen intención informacional y la otra mitad se la reparten entre las navegacionales y las transaccionales, con algo más de frecuencia de estas últimas. En el caso de las multimedia, hemos considerado una muestra mucho menor dado que se trata de una intención muy clara y las páginas de resultados apenas presentan variedad en la forma de presentar las imágenes.

En base a esto se han repartido las 22 tareas entre los 58 usuarios de forma que cada uno realizara 7 tareas. Se llevó a cabo un total de 406 tareas: 216 con intención informacional, 58 de tipo navegacional, 74 transaccionales y 58 multimedia. Aproximadamente la mitad de las tareas se realizaron en Google y la otra mitad en Yahoo!

Las temáticas elegidas fueron generales, así se evitaba un sesgo por especialidades. En la tabla 1 se muestra un ejemplo de tarea para cada tipo de consulta.

\subsection{Variables}

Las variables independientes han sido:

- Tipos de consultas: informacionales, navegacionales, transaccionales y multimedia.

- Tipos de resultados: orgánicos y patrocinados.

- AoIs (áreas de interés): títulos, snippets, url e imágenes.

Las variables dependientes son las fijaciones en cada AoI considerando dos de las métricas más utilizadas en estudios de eye tracking (Poole, 2004; Granka, Feusner y Lorigo, 2008; Nielsen y Pernice, 2009):

- El número de fijaciones en cada área: número de veces que los usuarios fijaron la vista en cada una de las áreas mencionadas.

- La duración de las fijaciones en cada área: tiempo (milisegundos), que permanecieron con la mirada fijada en cada AoI.

\subsection{Preparación del software de eye tracking}

Para realizar este estudio se ha dispuesto del equipo de eye tracker Tobii T120, y del software Tobii studio enterprise edition, versión 1.2, ambos cedidos temporalmente por Tobii Inc. a través de la empresa Alt 64.

Las tareas que los participantes debían resolver usando un buscador se les dieron ya tecleadas en Google y Yahoo! Las páginas de resultados se habían guardado previamente, de forma que todos accedieron a las mismas SERPs, lo que hace que sean comparables los resultados.

Las AoIs marcadas contemplan las dos grandes áreas: resultados orgánicos y enlaces patrocinados, subdividida esta última área a su vez en zona superior y lateral. Para cada zona de la pantalla se han marcado los tres primeros resultados por ser los que reciben la gran mayoría de las miradas (Hotchkiss, Alston, Edwards, 2006) (figura 2).

\begin{tabular}{|l|l|l|}
\hline \multicolumn{1}{|c|}{ Intención } & \multicolumn{1}{c|}{ Enunciado } & \multicolumn{1}{c|}{ Consulta } \\
\hline Informacional & ¿Qué horario tiene el museo del Louvre en París? & horario louvre \\
\hline Navegacional & Busque la página web oficial de la cadena Televisión Española & televisión española \\
\hline Transaccional & $\begin{array}{l}\text { Encuentre una página web donde pueda reservar una mesa en un } \\
\text { restaurante céntrico de Barcelona }\end{array}$ & restaurante barcelona \\
\hline Multimedia & Encuentre una imagen de la Alhambra al atardecer & alhambra atardecer \\
\hline
\end{tabular}

Tabla 1. Ejemplos de tareas para distintos tipos de intenciones 


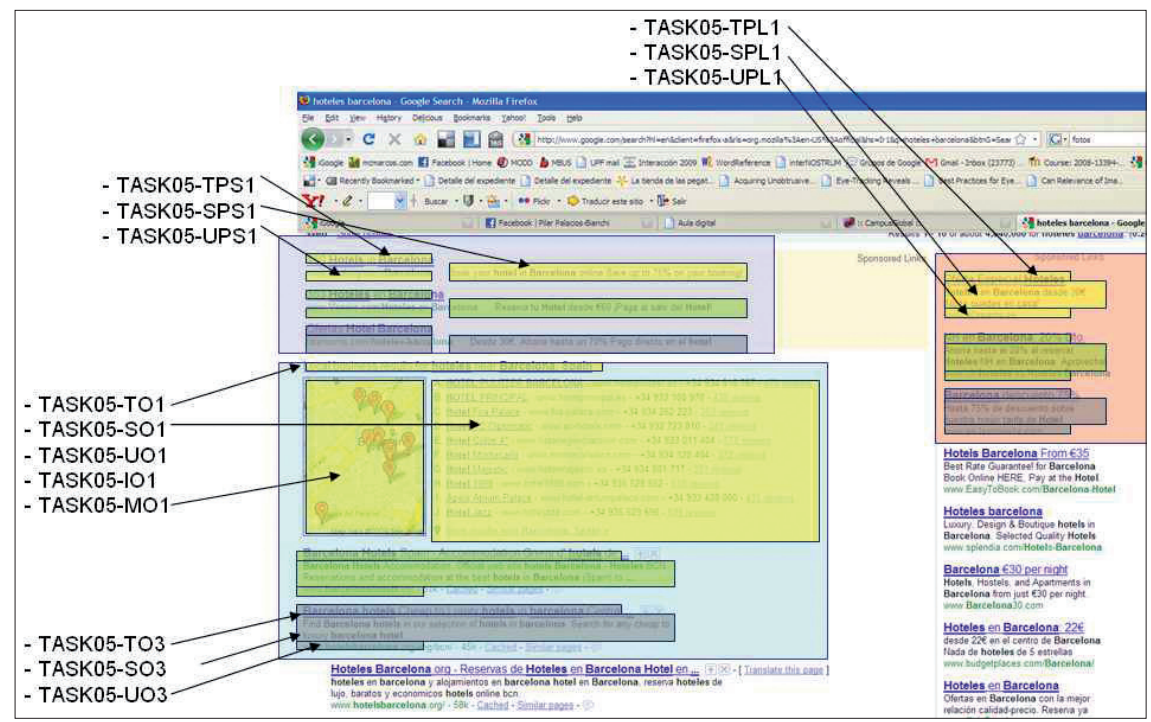

Figura 2. Ejemplo de marcado de Aols para resultados orgánicos y enlaces patrocinados en una SERP

\subsection{Desarrollo de las sesiones: encuestas y pruebas}

Tras recibir a cada participante y presentarle el experimento se le pidió que respondiera una encuesta con datos demográficos y de uso de internet. Después se procedió a calibrar el monitor para asegurar el correcto seguimiento de los ojos del usuario. La calibración fue correcta para los 58 usuarios. A continuación se realizaron las 7 tareas de forma consecutiva. Cada participante revisaba los resultados y clicaba el que creía que respondía mejor a lo que buscaba, lo que ponía fin a la tarea de búsqueda y se pasaba a la siguiente consulta.

El orden en que se les han presentado a los usuarios las tareas se ha ido alternando para evitar sesgos, debido a la familiaridad que pudieran tomar con la sala del laboratorio, los nervios de los primeros minutos, el cansancio o la prisa del final.

No todas las búsquedas han presentado enlaces patrocinados, y cuando lo han hecho no siempre ha sido en la zona superior y en la lateral, ni con al menos 3 enlaces en cada una, por lo que el número de éstos ha sido muy inferior que el de resultados orgánicos. Tampoco se han encontrado muchos resultados que incluyeran imágenes o mapas.

\section{Resultados}

\subsection{Resultados orgánicos y enla-} ces patrocinados

Del total de SERPs analizadas, sólo un 39\% presentaron enlaces patrocinados. Este porcentaje se reparte de la siguiente forma: $36 \%$ en consultas informacionales, $43 \%$ navegacionales, $76 \%$ transaccionales y $0 \%$ en consultas multimedia, lo que supone $77,25,56$ y 0 páginas respectivamente.

Hemos tenido en cuenta estos datos al comparar las fijaciones entre resultados orgánicos y patrocinados (tabla 2), ya que si no se presentan enlaces publicitarios no es posible registrar fijaciones en ellos. Por ese mismo motivo hemos eliminado para este análisis las consultas multimedia.

Si las cifras de la tabla 2 ya muestran claramente que los resultados orgánicos concentran la mayor parte de la atención del usuario en todos los tipos de consultas, la figura 3 lo refleja en forma de porcentajes, y se aprecia que las consultas que mayor número de fijaciones [y con más duración] registraron en los enlaces orgánicos fueron las informacionales, seguidas por las navegacionales y las transaccionales.

Esta distribución de la atención del usuario en los resultados orgánicos sugiere que las consultas informacionales son las que demandan un mayor análisis de los resultados incluidos en la SERP: el usuario requiere un mayor número de fijaciones y mayor tiempo para decidir qué resultados se adecuan a la consulta planteada al buscador.

En las de tipo navegacional y transaccional, la revisión de la SERP es más directa y requiere menos tiempo de evaluación de los resultados. En ellas el número de fijaciones y el tiempo invertido es menor, lo que hace que las zonas en las que los usuarios concentran

\begin{tabular}{|l|c|c|c|c|}
\hline & \multicolumn{2}{|c|}{ Orgánicos } & \multicolumn{2}{c|}{ Patrocinados } \\
\hline & $\begin{array}{c}\text { Promedio de } \\
\text { fijaciones por } \\
\text { tarea }\end{array}$ & $\begin{array}{c}\text { Duración (ms) } \\
\text { de fijaciones por } \\
\text { tarea }\end{array}$ & $\begin{array}{c}\text { Promedio de } \\
\text { fijaciones por } \\
\text { tarea }\end{array}$ & $\begin{array}{c}\text { Duración (ms) } \\
\text { de fijaciones por } \\
\text { tarea }\end{array}$ \\
\hline Informacional & 8,53 & $5.814,64$ & 0,64 & 446,95 \\
\hline Navegacional & 5,84 & $4.757,24$ & 1,12 & 530,24 \\
\hline Transaccional & 4,05 & $2.840,22$ & 2,20 & $1.504,55$ \\
\hline Promedio & $\mathbf{6 , 1 4}$ & $\mathbf{4 . 4 7 0 , 7 0}$ & $\mathbf{1 , 3 2}$ & $\mathbf{8 2 7 , 2 4}$ \\
\hline $\begin{array}{l}\text { Promedio de duración } \\
\text { por cada fijación }\end{array}$ & \multicolumn{2}{|c|}{728,13 ms por fijación } & 626,70 ms por fijación \\
\hline
\end{tabular}

Tabla 2. Promedio del número de fijaciones en las Aols y su duración en las zonas de resultados orgánicos y de enlaces patrocinados. Se ha calculado el promedio para cada tarea. 
su atención cobren más importancia a la hora de identificar las características que influyen en el proceso de selección de resultados.

Por su parte, la atención prestada a los enlaces patrocinados, es sustancialmente menor que a los resultados orgánicos. En las consultas con intención transaccional se observa una mayor relevancia de los anuncios, y estas consultas son las únicas que muestran un número de fijaciones más similar entre resultados orgánicos y patrocinados, lo que indica que hay un interés manifiesto de los usuarios en este tipo de resultados cuando sus consultas involucran la realización de acciones (comprar, descargar...) y no sólo la obtención de información. Por el contrario, en las consultas informacionales, que fueron las que mayor número de fijaciones dieron en los resultados orgánicos, los usuarios apenas prestan atención a los enlaces patrocinados, y ocurre algo similar con las navegacionales.

La tendencia es clara: si la intención es obtener información de algún tema en particular o llegar a un sitio web en concreto, los resultados orgánicos son los más revisados. En cambio, si la intención involucra la realización de algún tipo de acción los resultados patrocinados son mucho más tenidos en cuenta.

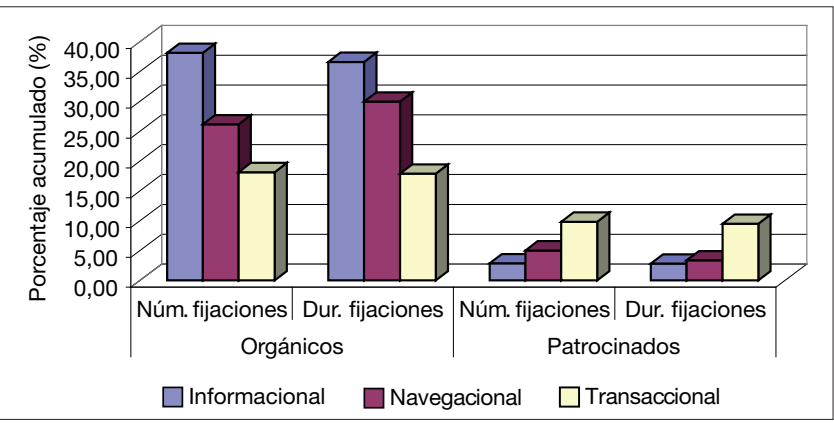

Figura 3. Porcentaje promedio de fijaciones y de duración de éstas en resultados orgánicos y enlaces patrocinados para las distintas intenciones de búsqueda

A continuación estudiamos cada tipo de consulta en función de la intención que hay tras ella y lo hacemos analizando las fijaciones en cada área de los resultados orgánicos, ya que son éstos los que concentran la mayoría del tiempo la atención de los usuarios. Sólo en el caso de las intenciones transaccionales se analizarán los datos obtenidos para los enlaces patrocinados.

\section{"Si la intención es obtener información o llegar a un sitio web concreto, los resultados orgánicos son los más revisados"}

\subsection{Consultas con intención informacional}

El comportamiento de los usuarios en una búsqueda de tipo informacional es bastante definido. Se fijan principalmente en el snippet intentando decidir si ese resultado concuerda con la información que están buscando. Esta evaluación es complementada por el título, que también ofrece una idea importante del contenido.

\section{"Los enlaces patrocinados se tienen en cuenta sólo si la intención es realizar una acción"}

Si tenemos en cuenta el tipo de información que los usuarios desean encontrar en este tipo de búsqueda (por ejemplo "¿Cuáles son los principales puntos turísticos de Granada?") el número de fijaciones en las distintas áreas y la duración de éstas son datos bastante equilibrados (figura 4).

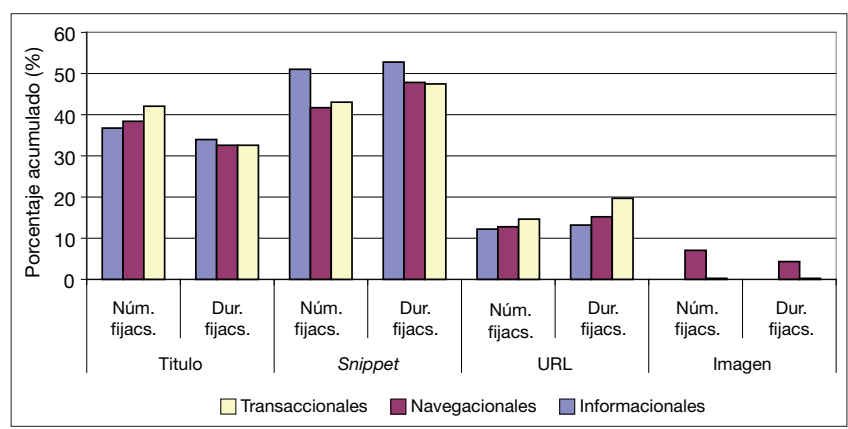

Figura 4. Número de fijaciones y duración de éstas en resultados orgánicos. Se presentan divididas por área de interés para búsquedas con intención informacional, navegacional y transaccional

En las consultas informacionales se confirma la relevancia del snippet (53\% del tiempo de las fijaciones), seguido de cerca del título (34\%) y en menor medida el url (13\%). En este caso las imágenes resultan ignoradas, algo que se debe en gran medida a que han aparecido pocas veces, por lo que el número de fijaciones y su duración es casi nulo en comparación con las otras AoIs.

Otras formas de ver estos resultados son: el mapa de calor generado por eye tracking (figura 5, parte superior), donde se observa cuáles fueron los puntos en los que los usuarios concentraron su atención; y el mapa llamado gazeplot en el que se aprecia el orden en el que varios usuarios miraron la pantalla y las zonas donde fijaron la atención durante más tiempo (figura 5, parte inferior).

Estos resultados sugieren que un buscador debería orientar su estrategia de despliegue de resultados para 




Figura 5. Mapas de calor y gazeplot para tres consultas con distinta intención

búsquedas de tipo informacional hacia una buena presentación de la descripción de sus páginas (snippets) así como a los títulos. Además, los anuncios que aparecen junto a los resultados no parecen tener gran impacto y en cambio ocupan un espacio que puede ser tenido en cuenta para una mejor organización de la información en la interfaz del buscador.

También los autores de páginas web deben tener en cuenta la información que ofrecen en la etiqueta meta "description", ya que los buscadores Google y Yahoo! toman el snippet de ella si detectan que los términos de búsqueda aparecen ahí.

\subsection{Consultas con intención navegacional}

A diferencia de las búsquedas de tipo informacional, donde el usuario podría estar interesado en múltiples sitios siempre y cuando éstos se relacionen con sus intereses informacionales, las búsquedas navegacionales tienen la preintención por parte del usuario de visitar un sitio concreto.

En estos casos, más que analizar si un resultado concuerda con un tema en particular, lo que el usuario quiere es saber si alguno de los resultados obtenidos corresponde al sitio que tiene en mente. Por este motivo las fijaciones en las AoIs varían con respecto a las búsquedas de tipo informacional. Si bien los tres principales puntos de atención se siguen manteniendo (snippet, título y url) (figura 4), se nota un aumento de atención en el título (se mira un $38 \%$ de las veces y se mantiene la atención en él en un $33 \%$ del tiempo) y en el url, dos áreas que permiten una identificación más directa de la página web. En estos casos, aunque el snippet continúa siendo el área observada durante más tiempo (48\%), ya no es la que se mira más veces $(42 \%$ frente al $51 \%$ de las veces que se ha observado el título). El motivo de que el snippet obtenga mayor tiempo de observación seguramente se debe a que es más extenso, por lo que necesita más tiempo para ser leído. Resulta interesante observar cómo el título en estos casos se mira más veces que el snippet, aunque menos tiempo puesto que es más breve.

Este resultado se puede poner en relación con el obtenido por Cutrell y Guan (2007b), quienes descubrieron que un snippet más extenso beneficia al usuario en las consultas informacionales pero no en las navegacionales, puesto que cuanto más espacio ocupen los snippets en pantalla menor número de resultados se podrán mostrar, y al mismo tiempo la información irrelevante estará llenando un espacio mayor. 
Aunque no son una de las zonas más importantes, en este tipo de consultas se presta más atención a las imágenes.

También se observa ahora, aunque con muy poca relevancia, una mayor fijación en los enlaces patrocinados (figura 3), que reciben el 5\% de las fijaciones totales, casi el doble que lo obtenido en las consultas informacionales. Esto puede estar relacionado con la especificidad de la consulta navegacional: el usuario quiere encontrar un sitio determinado y a veces este sitio puede estar listado dentro de los anuncios publicitaros.

\section{"Existe una relación entre la intencionalidad del usuario y la forma de mirar la pantalla"}

\subsection{Consultas con intención transaccional}

El análisis de la distribución de fijaciones de los usuarios en las SERPs para estas consultas ofrece un panorama interesante. No sólo se confirma el manifiesto interés de los usuarios en los enlaces patrocinados sino que se puede especificar con más detalle en qué AoIs.

Lo primero a tener en cuenta es que la zona que atrae la mayor parte de las miradas sigue siendo la de resultados orgánicos ( $82 \%$ por $17 \%$ los patrocinados), y dentro de éstos se mantiene el orden de atención en las áreas de interés: snippet, título y url. Esto refleja que independientemente de su intención, el usuario presta mayor atención a esta zona por ser la que ofrece una descripción más completa de los resultados. El hecho de que el número de fijaciones recibidas por un AoI y su duración promedio no siempre coincidan se debe en buena parte al espacio que ocupan en pantalla. Por ejemplo, los títulos se llevan el $38 \%$ de las fijaciones y el $28 \%$ del tiempo de observación: al ocupar menos espacio es necesario menor tiempo de observación para leerlo. Ocurre exactamente lo contrario con los snippets, que son más largos y por tanto el porcentaje que aparece como resultado de los promedios de duración de las fijaciones en principio no extraña que sea mayor que el porcentaje de veces que han sido mirados: $50 \%$ de veces se han mirado, ocupando un 56\% del tiempo de fijación.

La atención sigue siendo mayor (más del doble) en los resultados orgánicos que en los patrocinados, pero el número de fijaciones es muy superior en las consultas transaccionales que en el resto $(9,8 \%$ frente a $2,8 \%$ en informacionales y $5 \%$ en navegacionales), por lo que merece la pena analizarlas con más detenimiento.

Se han estudiado las dos posiciones de enlaces patrocinados: la zona superior de la pantalla, antes de los resultados orgánicos, y la parte lateral derecha. De las 406 SERPs estudiadas en este proyecto hubo 158 que presentaron enlaces patrocinados. De ellas, todas los tenían en la zona lateral y sólo un tercio de éstas presentaban además anuncios en la zona superior. A pesar de ser pocos, llama la atención que el 79\% de las fijaciones se dieron en la zona de anuncios superior frente al $21 \%$ que fueron a la zona lateral. La figura 6 apoya estos resultados con los mapas obtenidos para 4 usuarios en dos SERPs.
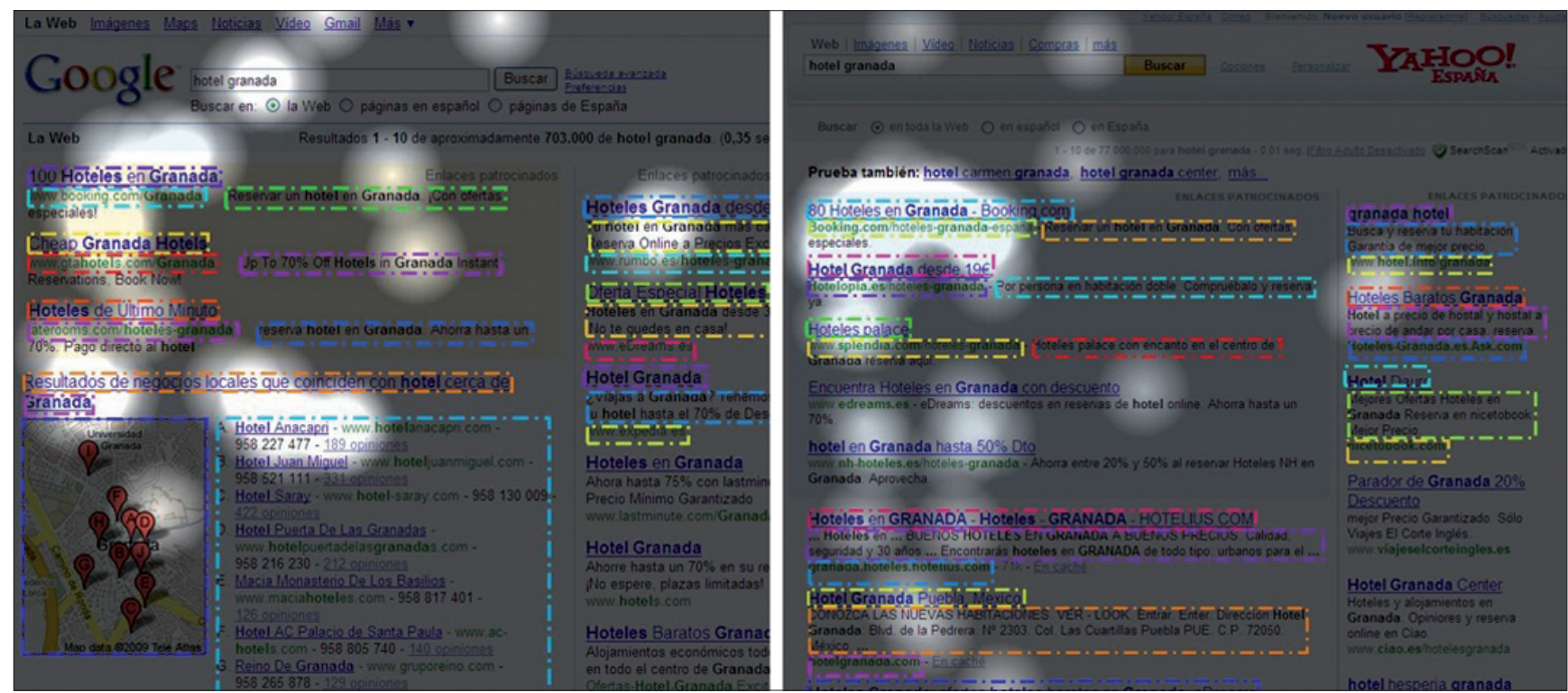

Figura 6. Mapa de tipo gaze opacity en el que se muestran las zonas que más vieron los usuarios en dos SERPs para consultas con intención transaccional 
De las tres AoIs que estudiamos en los enlaces patrocinados observamos que el título concentra la mayoría del tiempo de observación (43\%), mientras que el snippet y el url se reparten casi a partes iguales el resto (28 y $29 \%$, respectivamente, figura 7 ).

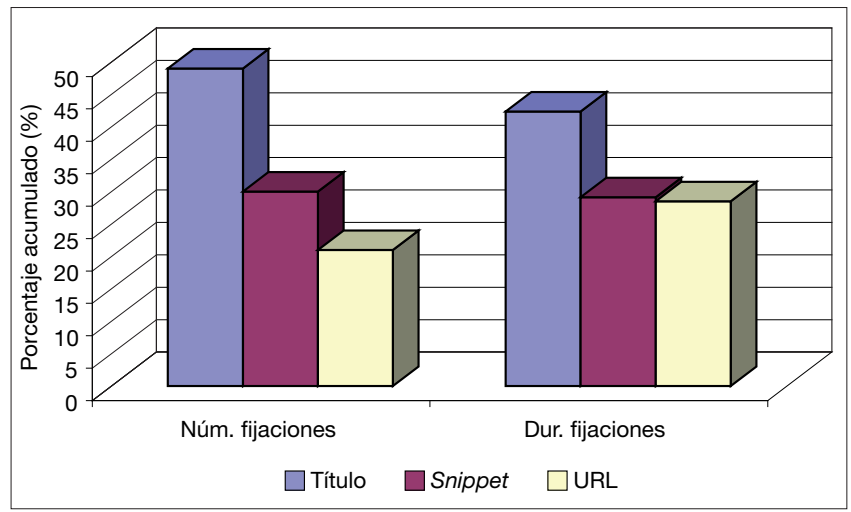

Figura 7. Número de fijaciones y su duración en las tres Aols: título, snippet $y$ url de los enlaces patrocinados en consultas transaccionales

Estos porcentajes llaman la atención si los comparamos con lo que ocurría en las consultas informacionales y navegacionales: cuando se trataba de resultados orgánicos, el snippet era siempre visto más veces y con mayor duración que el título. Es interesante ver cómo el url tiene mucho más peso aquí que en los resultados orgánicos, aunque sin llegar a superar al snippet en número de fijaciones y duración de éstas.

\subsection{Consultas multimedia}

En este tipo de búsquedas los usuarios presentan un comportamiento muy parejo: la gran mayoría de las fijaciones las reciben las imágenes. Esto ocurre por primera vez con referencia a los otros tres tipos de búsquedas: el snippet deja de ser el área más relevante y deja paso al elemento gráfico, manteniéndose a una buena distancia tanto si atendemos al número de fijaciones (76\%) como a su duración promedio (71\% del tiempo) (figura 4).

Si se observa el mapa de recorrido e intensidad de la mirada para una búsqueda de tipo multimedia (figura 8 ) se pueden entender mejor los resultados mostrados en la figura 4. La forma en que los resultados son organizados en la pantalla explica la atención inmediata de los usuarios en las imágenes. Adicionalmente hay que considerar que cuando el usuario realiza estas consultas está anunciando con anterioridad que está interesado en este tipo de resultados con lo que las descripciones textuales pasan a un segundo plano.

\section{"Los enlaces patrocinados no suscitan interés en los usuarios cuya intención de búsqueda no es transaccional"}

Por otra parte, los usuarios se fijan principalmente en la primera fila de resultados, con lo que estas imágenes son generalmente las únicas que el usuario llega a evaluar.

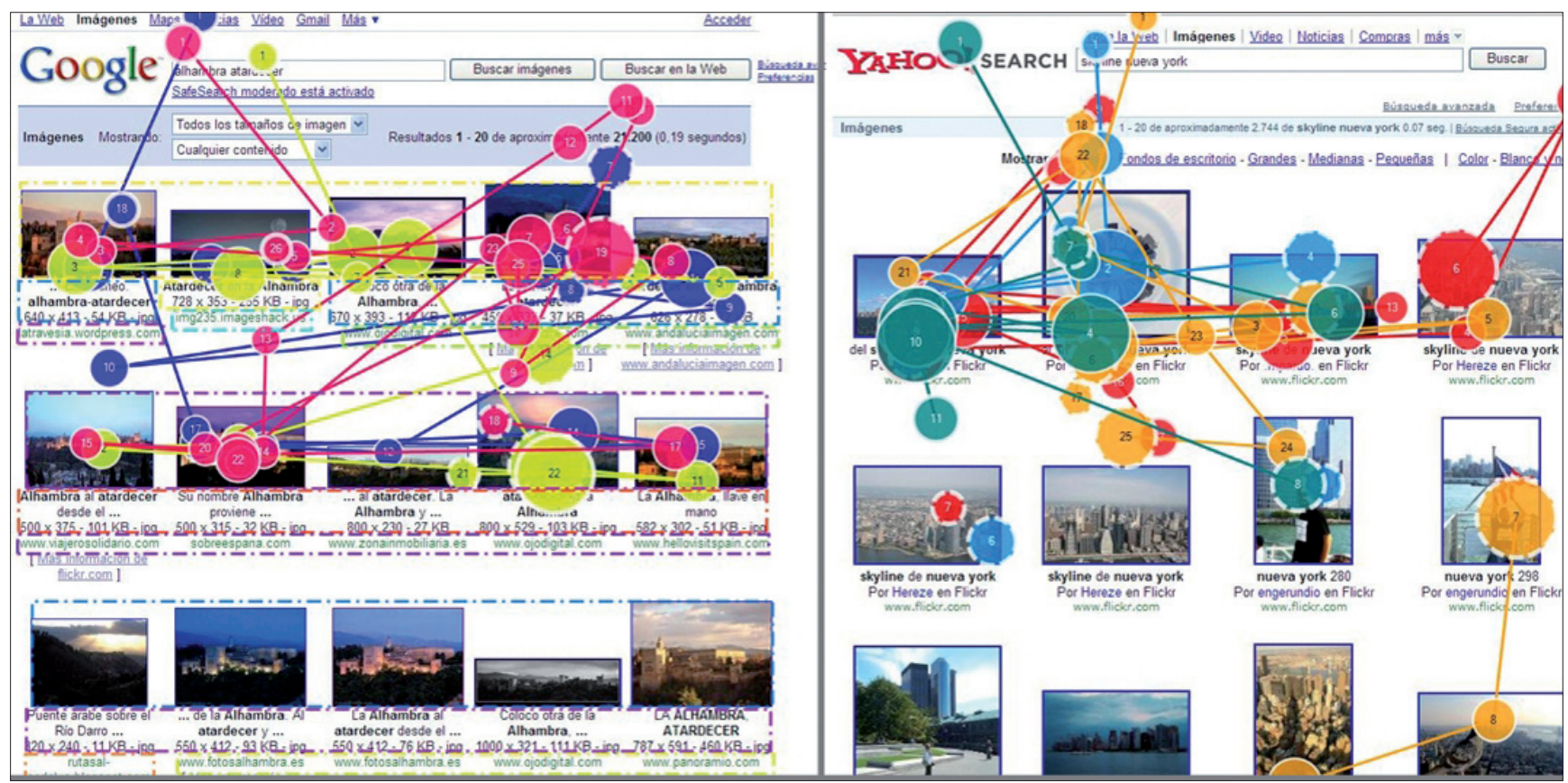

Figura 8. Gazeplot para consultas multimedia en Google y Yahoo! 


\section{Conclusiones}

Una de las preguntas que nos hacíamos al plantear este estudio era qué información de los resultados obtenía mayor atención por los usuarios. Para ello hemos estudiado los resultados en función de tres variables: el tipo (orgánicos y patrocinados); las áreas que los componen (título, snippet, url, y en ocasiones imagen) y la intención que implica la consulta (informacional, navegacional, transaccional y multimedia).

Cruzando los datos de tipo y áreas de interés hemos descubierto que en los resultados orgánicos los usuarios se fijaron casi la mitad del tiempo en el snippet (44\%), seguido del título (39\%) y del url (17\%) (figura 4). En cambio en los enlaces patrocinados los usuarios mostraron más interés por el título (43\%), seguido del snippet (28\%)y el url (29\%) (figura 7 ).

Añadiendo a esta información la variable de la intención de la consulta, hemos obtenido que para las consultas con intención informacional, navegacional y transaccional, el área más observada es el snippet, seguida de cerca por el título en los resultados orgánicos. En las consultas con intención transaccional, los enlaces patrocinados reciben un número no despreciable de fijaciones, sobre todo su título, seguido por el snippet y el url a partes iguales. Por último, en las consultas multimedia la imagen se lleva la mayor parte de las miradas.

\section{"Los anuncios situados en la zona superior reciben muchas más miradas que los que se encuentran en el lateral"}

\section{Estos resultados nos llevan a afirmar que:}

Los enlaces patrocinados no suscitan interés en los usuarios cuya intención de búsqueda no es transaccional; y al revés, les prestan atención cuando hay intención transaccional, por ejemplo las búsquedas con una finalidad comercial. Los anunciantes deberán optimizar sus anuncios para aparecer sobre todo asociados a consultas con intención transaccional. Deberán cuidar en especial el título, dado que es la zona del anuncio que más se observa. En los anuncios el url recibe una notable atención, así que será recomendable utilizar urls amigables, fáciles de recordar y cortas. Es de destacar que los anuncios situados en la zona superior han recibido muchas más miradas que los que se encuentran en el lateral. Posiblemente se debe a que los de la zona superior se confunden más fácilmente con resultados orgánicos mientras que los usuarios no miran tanto al lateral porque tienen más claro que es una zona donde suele haber publicidad.

El fragmento de texto o snippet es el área de resultados que más leen los usuarios. Esperan encontrar aquí la información que les ayudará a decidir si clican en el resultado. Es cierto que es el área más extensa y eso favorece que la mirada se dirija en más ocasiones a ella y que la duración sea mayor, pues leerla requiere más tiempo que las demás. Deberá contener la información que mejor responda a la consulta planteada, de manera que sirva para decidir si la página a la que apunta es o no de interés para el usuario.

El título es un área que debe concentrar mucha información en pocas palabras y que a pesar de su brevedad recibe buena parte del tiempo de observación de los usuarios. Su redacción debe estar muy pensada y cuidada para que refleje bien el contenido de la página.

Por último, el url recibe una observación menor que el resto de áreas, pero se trata de una información interesante porque permite conocer si el resultado apunta a un sitio web fiable, en el caso de las informacionales y transaccionales, y a un sitio oficial en el caso de las navegacionales.

\section{"El fragmento de texto o snippet es el área de resultados que leen más los usuarios"}

Estas conclusiones apoyadas en los resultados obtenidos son nuestra aportación al campo de estudio del user search behavior en la línea de Broder (2002), Rose y Levinston (2004) y Lee, Liu y Cho (2005). Finalmente se puede señalar que sería un gran avance poder interpretar de forma automática las consultas para detectar si el usuario busca cierta información, una página web en particular o desea realizar alguna transacción (Baeza-Yates, Calderón-Benavides y González-Caro, 2006), o incluso llegar a detectar si busca un dato concreto, informaciones contrastadas u opiniones (Baeza-Yates, Calderón-Benavides; González-Caro y Marcos, 2008).

\section{Referencias}

Aula, Anne; Majaranta, Päivi; Räihä, Kari-Jouko. "Eye-tracking reveals the personal styles for search result evaluation". En: Procs of human-computer interaction - Interact, 2005, pp. 1058-1061.

Baeza-Yates, Ricardo; Calderón-Benavides, Maritza L.; González Caro, Cristina N. "The intentions behind web queries". En: Lecture notes in computer science, v. 4209. Heidelberg: Springer, 2006, pp. 99-108.

Baeza-Yates, Ricardo; Calderón-Benavides, Liliana; González-Caro, Cristina; Marcos, Mari-Carmen. “Personas o máquinas? Servicios 
de preguntas y respuestas vs. buscadores web". En: Jornades catalanes d'informació i documentació (Barcelona, 22-23 de mayo de 2008). Barcelona: Cobdc, 2008.

Broder, Andrei. "A taxonomy of web search". En: ACM Sigir forum, 2002, v. 36, n. 2 , pp. $3-10$

Codina, Lluís; Marcos, Mari-Carmen. "Posicionamiento web: conceptos y herramientas". El profesional de la información, 2005, marzo-abril, v. 14 n. 2, pp. 84-99.

http://www.elprofesionaldelainformacion.com/contenidos/2005/marzo/1.pdf

Cutrell, Edward; Guan, Zhiwei. "What are you looking for?: An eye-tracking study of information usage in web search". En: Procs of the Sigchi conf on human factors in computing systems. New York: ACM Press, 2007.

GEA Internet Project Consulting. Eyetracking search marketing: Estudio de search marketing orientado a turismo realizado con técnicas de eyetracking, 2009.

http://www.geaipc.com/blog/estudios-best-practices/eyetracking-searchmarketing/

Granka, Laura; Feusner, Matthew; Lorigo, Lori. "Eye monitoring in online search". En: Hammoud, R. (ed.). Passive eye monitoring: algorithms, applications and experiments. Springer-Verlag, 2008, pp. 283-304

Granka, Laura; Joachims, Thorsten; Gay, Geri. "Eye-tracking analysis of user behavior in WWW search". En: Procs of the 27th Annual international ACM SIGIR Conference on Research and Development in information Retrieval (Sheffield, United Kingdom, July 25 - 29, 2004). New York: ACM Press, 2004, pp. 478-479.

Guan, Zhiwei; Cutrell, Edward. "An eye tracking study of the effect of target rank on Web search". En: Procs of the Sigchi conf on human factors in computing systems. New York: ACM Press, 2007.

Hotchkiss, Gord; Alston, Steve; Edwards, Greg. Eye tracking report: Google, MSN and Yahoo! compared. 2006.

http://www.enquiro.com/eyetrackingreport.asp

Kaushik, Avinash. Web analytics: an hour a day. New York: John Wiley \& Sons, 2007.

Kaushik, Avinash. Web analytics 2.0. New York: John Wiley \& Sons, 2009

Lee, Uichin; Liu, Zhenyu; Cho, Junghoo. "Automatic identification of user goals in web search". En: Fourteenth intl World Wide Web conf. New York: ACM Press, 2005, pp. 391-400.

Lorigo, Lori; Haridasan, Maya; Brynjarsdóttir, Hrönn; Xia, Ling Joachims, Thorsten; Gay, Geri; Granka, Laura; Pellacini, Fabio; Pan, Bing. "Eye tracking and online search: Lessons learned and challenges ahead". Journal of the American Society for Information Science and Tech nology, 2008, v. 59, n. 7, pp. 1041-1052.
Nielsen, Jakob; Pernice, Kara. Eyetracking web usability. Berkeley: New Riders Press, 2009.

Poole, Alex; Ball, Linden J. "Eye tracking in human-computer interaction and usability research: current status and future prospects". En: Ghaoui, Claude (Ed.). Encyclopedia of human computer interaction. Idea Group. http://www.alexpoole.info/academic/Poole \&Ball\%20EyeTracking.pdf

RealEyes. Why you need 50 people in eye tracking studies. 2007 http://www.realeyesit.com/press/why-you-need-50-people-in-eye-trackingstudies.aspx

Rele, Rachana S.; Duchowski, Andrew T. "Using eye tracking to evaluate alternative search results interfaces". En: Procs of the Human Factors and Ergonomics Society, Sept 26-30, 2005, Orlando, FL, HFES, 2005. http://andrewd.ces.clemson.edu/research/vislab/docs/Final_HFES_Search. $p d f$

Rose, Daniel E.; Levinson, Danny. "Understanding user goals in Web search". En: Thirteenth intl World Wide Web conf. New York: ACM Press, 2004, pp. 13-19.

Terai, Hitoshi; Saito, Hitomi; Egusa, Yuka; Takaku, Masao; Miwa, Makiko; Kando, Noriko. "Differences between informational and transactional tasks in information seeking on the web". En: Procs of the $2^{\text {nd }}$ int symposium on information interaction in context table of contents. ACM Intl conf proc series, 2008, v. 348, pp. 152-159.

Tobii StudioTM. Tobii StudioTM 1.2 product description. 2009 http://www.tobii.com/archive/files/19202/Tobii +Studio+1_2+Product+ Description.pdf.aspx

Van Gompel, Roger P. G.; Fischer, Martin H.; Murray, Wayne S.; Hill, Robin L. "Eye-movement research: An overview of current and past developments". En: Van Gompel, Roger P. G.; Fischer, Martin H.; Murray, Wayne S.; Hill, Robin L. (eds.). Eye movements: A window on mind and brain. Oxford: Elsevier, 2007, pp. 1-28.

Mari-Carmen Marcos, Universitat Pompeu Fabra, Departamento de Comunicación. Roc Boronat 138, 08018 Barcelona, Spain. mcarmen.marcos@upf.edu

Cristina González-Caro, Universitat Pompeu Fabra, Departamento de Tecnologías de la Información y las Comunicaciones. Tanger 122-140, 08018 Barcelona, Spain.

cristina.gonzalez@upf.edu

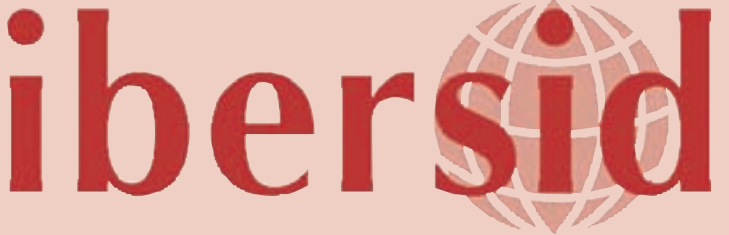

Zaragoza, 4-7 de octubre de 2010

\section{http://www.ibersid.org}

IBERSID es un foro internacional anual nacido en 1996 con el objetivo de promover la investigación entre académicos, investigadores y estudiantes de las Ciencias de la Documentación y de otras disciplinas relacionadas, interesados en identificar, analizar y discutir de forma rigurosa, interdisciplinar, abierta y distendida los problemas profesionales $y$ científicos.

La discusión se concentra en torno al concepto de sistemas de información y documentación como núcleo común alrededor del cual los distintos especialistas -archiveros, bibliógrafos, bibliotecarios, documentalistas, museólogos, gestores de la información y del conocimiento, etc.- pueden dialogar. 